\title{
Lifetime Measurements of GaAs photocathodes at the Upgraded Injector Test Facility at Jefferson Lab*
}

\author{
M. Herbert ${ }^{\dagger a}$, J. Enders ${ }^{a}$, M. Poelker ${ }^{b}$, C. Hernandez-Garcia ${ }^{b}$ \\ ${ }^{a}$ Institut für Kernphysik, Technische Universität Darmstadt, Darmstadt, Germany \\ ${ }^{b}$ Thomas Jefferson National Accelerator Facility, Newport News, VA, United States of America \\ E-mail: mherberteikp.tu-darmstadt.de
}

\begin{abstract}
Photo-cathodes based on GaAs can be used in photo-electron sources to supply spin-polarized, high-current electron beams for various applications. An activation, adding a thin surface layer, is needed to achieve negative electron affinity (NEA) for such cathodes. Typically, Cs is used in combination with an oxidant. Previous studies have suggested that the addition of $\mathrm{Li}$ to this process can increase the quantum efficiency $\eta$ of the cathode as well as the lifetime $\tau$ of the cathode surface layer, both crucial parameters for photo-electron source operation. Charge lifetime studies of bulk GaAs photo-cathodes activated with $\mathrm{Cs}, \mathrm{NF}_{3}$, and $\mathrm{Li}$ have been conducted using the photo-electron gun of the Upgraded Injector Test Facility (UITF) at the Thomas Jefferson National Accelerator Facility (JLab), extracting beam currents of up to $100 \mu \mathrm{A}$. Evidence for significant charge lifetime enhancement with $\mathrm{Li}$ in the activation process is found.
\end{abstract}

The 18th International Workshop on Polarized Sources, Targets, and Polarimetry, PSTP2019

23-27 September, 2019

Knoxville, Tennessee

*Work supported by DFG (GRK 2128 “AccelencE”), BMBF (05H18RDRB1), and through the Helmholtz Graduate School for Hadron and Ion Research for FAIR, and by Jefferson Science Associates, LLC under U.S. DOE Contract No. DE-AC05-06OR23177

†Speaker. 


\section{Introduction}

Electron sources providing a high degree of polarization and high beam current are in demand for a multitude of accelerator applications, such as energy-recovery linacs (ERLs [1,2]), positron sources [3], colliders [4] and electron cooling at high-energy storage rings [5]. Promising candidates are based on state-of-the-art dircet-current (DC) high-voltage (HV) photo-emission guns, using photo-cathodes based on the III-V semiconductor gallium arsenide (GaAs).

The Thomas Jefferson National Accelerator Facility (JLab) has a long and successful history of operating GaAs-based polarized DC photo-guns at the Continuous Electron Beam Accelerator Facility (CEBAF [6-8]). An injector research and development setup, the Upgraded Injector Test Facility (UITF), is operated by the Center for Injectors and Sources (CIS) and features a $200 \mathrm{kV}$ DC photo-gun with adjacent $10 \mathrm{MeV}$ beamline for testing of injector components and experimental setups $[9,10]$. It constitutes a convenient environment for photo-cathode research. Comparably, albeit at a smaller scale, a dedicated test stand for DC photo-gun research and development, Photo-CATCH, with a $60 \mathrm{kV}$ photo-gun and $60 \mathrm{keV}$ beamline has been constructed at the Institut für Kernphysik of the Technische Universität Darmstadt. It provides an environment for research and development to optimize the existing DC photo-gun, which is located at the Spin-Polarized Injector SPIn of the in-house electron accelerator S-DALINAC [11-13]. A collaboration between the two institutes has been established in order to effectively capitalize on our joint area of research.

\section{Photo-electron emission from GaAs}

Effective photo-electron emission from GaAs cathodes requires a state of negative electron affinity (NEA), which is achieved through the application of a thin layer - typically a combination of caesium $(\mathrm{Cs})$ and an oxidant, e.g. oxygen $\left(\mathrm{O}_{2}\right)$ or nitrogen trifluoride $\left(\mathrm{NF}_{3}\right)$ - to the GaAs surface. Quality and composure of this layer determines the two main photo-cathode parameters: quantum efficiency $\eta$ and lifetime $\tau$, where $\eta$ characterizes the electron emission efficiency, i.e. how many electrons are emitted per incident photon, and is given by emitted photo-current $I_{p}$, incident laser power $P_{L}$ and wavelength $\lambda$ as [12]

$$
\eta(\lambda)=\left|\frac{h c_{0} I_{p}}{e P_{L} \lambda}\right| \cdot 100 \%
$$

where $h$ is Planck's constant, $c_{0}$ is the speed of light in vacuum, and $e$ is the elementary charge. The deterioration of the NEA layer with time is observable through a time-dependent decrease in $\eta$, which can be formalized as

$$
\eta(t)=\eta_{0} \cdot e^{-t / \tau}
$$

with a characteristic time constant or lifetime $\tau$. Different processes responsible for the decay are represented by individual lifetimes $\tau_{i}$. This correlation is commonly expressed as [14]

$$
\tau=\left(\sum_{i} \tau_{i}^{-1}\right)^{-1} .
$$

The two dominant causes of decay are i) the processes related to vacuum conditions, and ii) the processes related to extraction of beam through laser illumination and high-voltage application. 
The former is characterized by the so-called dark lifetime $\tau_{d}$, while the latter is characterized by the extracted charge $Q(t)=\int_{0}^{t} I\left(t^{\prime}\right) d t^{\prime}$, where $I(t)$ is the beam current, and a charge constant $k_{c}$. If the extracted current $I$ is constant, one can assume that the charge $k_{c}$ has been extracted once $\eta$ drops to $1 / \mathrm{e}$ of its initial value $\eta_{0}$ after a certain time, the so-called charge lifetime $\tau_{c}=I / k_{c}$. This leads to the two separate cases [15]

$$
\begin{aligned}
\eta(t) & =\eta_{0} \cdot e^{-t / \tau_{d}-Q(t) / k_{c}} & & \text { for } I \neq \text { const. } \quad, \quad \text { and } \\
& =\eta_{0} \cdot e^{-t \cdot\left(1 / \tau_{d}+1 / \tau_{c}\right)} & & \text { for } I=\text { const. } .
\end{aligned}
$$

For $I=$ const., $Q(t)$ is linear. For $I \neq$ const., $Q(t)$ can be represented using an exponential decay model

$$
Q(t)=Q_{t o t} \cdot\left(1-e^{-t / \tau_{c}}\right),
$$

where $Q_{t o t}=Q(t=\infty)$ is the total extracted charge. Then, the amount of charge extracted during viable operation $k_{c}$ can be estimated as $k_{c}=Q\left(\tau_{c}\right)=Q_{t o t} \cdot(1-1 / e)$.

Both high $\eta$ and long $\tau$ are desirable for high-current applications. It has been reported that introducing the alkali metal lithium $(\mathrm{Li})$ into the activation process with $\mathrm{Cs}$ and $\mathrm{NF}_{3}(\mathrm{GaAs}$ : $(\mathrm{Cs}, \mathrm{Li}$, $\left.\mathrm{NF}_{3}\right)$ ) can enhance the surface layer quality, hence increasing both $\eta$ and $\tau_{d}$ compared to the "standard" GaAs: $\left(\mathrm{Cs}, \mathrm{NF}_{3}\right)$ activation [16, 17]. Recent studies at Photo-CATCH on GaAs:(Cs, $\left.\mathrm{Li}, \mathrm{O}_{2}\right)$ yielded increased values for $\tau_{d}$, but did not report an increase in $\eta$ compared to the GaAs: $\left(\mathrm{Cs}, \mathrm{O}_{2}\right)$ process [12]. Recent collaborative efforts of our groups at JLab and TU Darmstadt have focused on investigations of $\tau_{c}$ for both GaAs: $\left(\mathrm{Cs}, \mathrm{Li}, \mathrm{NF}_{3}\right)$ and $\mathrm{GaAs}:\left(\mathrm{Cs}, \mathrm{Li}, \mathrm{O}_{2}\right)$, since the performance of such cathodes have not yet been evaluated during gun operation. First $\tau_{c}$ measurements for GaAs: $\left(\mathrm{Cs}, \mathrm{Li}, \mathrm{NF}_{3}\right)$ were conducted at UITF, with additional $\tau_{c}$ measurements for GaAs:(Cs, $\mathrm{Li}$, $\mathrm{O}_{2}$ ) planned at Photo-CATCH.

\section{Charge lifetime measurements at UITF}

\subsection{Setup}

For the measurements presented in this contribution, the low-energy source section of the UITF was used. Photo-cathodes are prepared in the activation chamber. It is equipped with a heater for surface cleaning, an anode ring for $I_{p}$ measurement, one dispenser for Cs and Li each, a valve for $\mathrm{NF}_{3}$ introduction, a movable mask with several openings of different size, and a window with attached diode laser system. Also, several manipulators are installed for translation and rotation of the cylindrical, cup-shaped photo-cathode mount or puck. The photo-cathode is placed on the front face of the puck, which can be mounted with its hollow back on the heater or on a manipulator. A seperate, adjacent chamber - connected to the activation chamber with a valve for photo-cathode transfer - houses the photo-gun, which has a $-200 \mathrm{kV}$ inverted-insulator geometry gun (IIGG) design that has been developed at CEBAF [18, 19] and features an anode ring. A reduced voltage of $-144 \mathrm{kV}$ was used due to incomplete $\mathrm{HV}$ conditioning of the gun at the time of the measurements. The adjacent beamline section used for the experiment is equipped with several steering and solenoid magnets, a Wien-filter for polarization control, and a dipole magnet. This magnet is situated at a bend that allows the cathode to be illuminated directly by the laser system, 
which is situated in a shielded box next to the beamline. An indircet online $P_{L}$ measurement is provided through a pick-off setup. The beam current $I$ was measured with a Faraday cup at the end of the beamline section.

\subsection{Procedure}

All measurements were conducted using the same bulk-GaAs photo-cathode sample. Prior to each activation, the photo-cathode was heat-cleaned in the activation chamber by ramping up the temperature to $600{ }^{\circ} \mathrm{C}$ over $1 \mathrm{~h}$ and maintaining this temperature for another $1 \mathrm{~h}$. The heating is then turned off, and the activation process is not started before the photo-cathode has cooled down to room temperature.

A $5 \mathrm{~mm}$ circular spot on the cathode surface was activated using a mask. The photoresponse was probed with $780 \mathrm{~nm},(1.1 \pm 0.1) \mathrm{mW}$ laser light, which was reduced during activation to $(26 \pm 1) \mu \mathrm{W}$ by inserting a filter. This was done to keep $I_{p}$ below $2.5 \mu \mathrm{A}$, in order to limit preliminary degradiation of the surface layer from charge extraction. A bias voltage of $233 \mathrm{~V}$ was applied to the anode ring during activation. $\mathrm{Cs}$ and $\mathrm{NF}_{3}$ were introduced using a standard Co-Deposition (Co-De) scheme: Cs is applied until a peak in $I_{p}$ is observed, with $\mathrm{NF}_{3}$ being introduced after $I_{p}$ drops to $75 \%$ of the peak current. $\mathrm{Cs}$ and $\mathrm{NF}_{3}$ flux is continued until $I_{p}$ reaches a plateau. The supply of both reagents is then stopped, starting with $\mathrm{NF}_{3}$. This scheme was enhanced by applying $20 \mathrm{~s}$ bursts of $\mathrm{Li}$ after $\mathrm{NF}_{3}$ deposition had started, until the bursts showed no increase in $I_{p}$. A total of 3 bursts were applied, as shown in fig. 1. This procedure was developed during previous studies at Photo-CATCH [20]. Two activations were conducted during the experiments, one for GaAs:(Cs, $\left.\mathrm{Li}, \mathrm{NF}_{3}\right)$, henceforth referred to as activation 1 , and one using a GaAs: $\left(\mathrm{Cs}, \mathrm{NF}_{3}\right)$ photocathode, henceforth referred to as activation 2.

The activated photo-cathode was then transferred to the gun chamber where a quantum efficiency scan was conducted in order to determine the coordinates of maximum $\eta$ within the activated area. This position was used for beam production with a laser wavelength of $780 \mathrm{~nm}$. Two lifetime runs were conducted: one using a GaAs: $\left(\mathrm{Cs}, \mathrm{Li}, \mathrm{NF}_{3}\right)$ photo-cathode, henceforth referred to as run 1, and one using a GaAs: $\left(\mathrm{Cs}, \mathrm{NF}_{3}\right)$ photocathode, henceforth referred to as run 2 . A constant $P_{L}$ was used during beam production because the feedback control system for auto-tuning $P_{L}$ in order to keep $I$ constant was not operational at the time of experiments. The initial beam current $I_{0}$ for run 1 was set to $(20 \pm 1) \mu \mathrm{A}$ with a corresponding initial laser power $P_{L, 0}$ of $(0.6 \pm 0.1) \mathrm{mW}$, before being increased to $(100 \pm 1) \mu \mathrm{A}$ with a corrseponding $P_{L, 0}$ of $(4.3 \pm 0.3) \mathrm{mW}$. Run 2 was started with $I=(100 \pm 1) \mu \mathrm{A}$ with a corrseponding $P_{L, 0}$ of $(3.8 \pm 0.1) \mathrm{mW}$. Pressure within the gun chamber was $<5 \times 10^{-11}$ mbar during experiments.

During the procedure, 3 different checkpoint values of $\eta$ were determined after finishing the activation sequence for later comparison: first within the activation chamber $\left(\eta_{i n i}\right)$, then after obtaining its maximum with a QE scan $\left(\eta_{\max }\right)$, and finally at the very beginning of beam extraction $\left(\eta_{0}\right)$. 


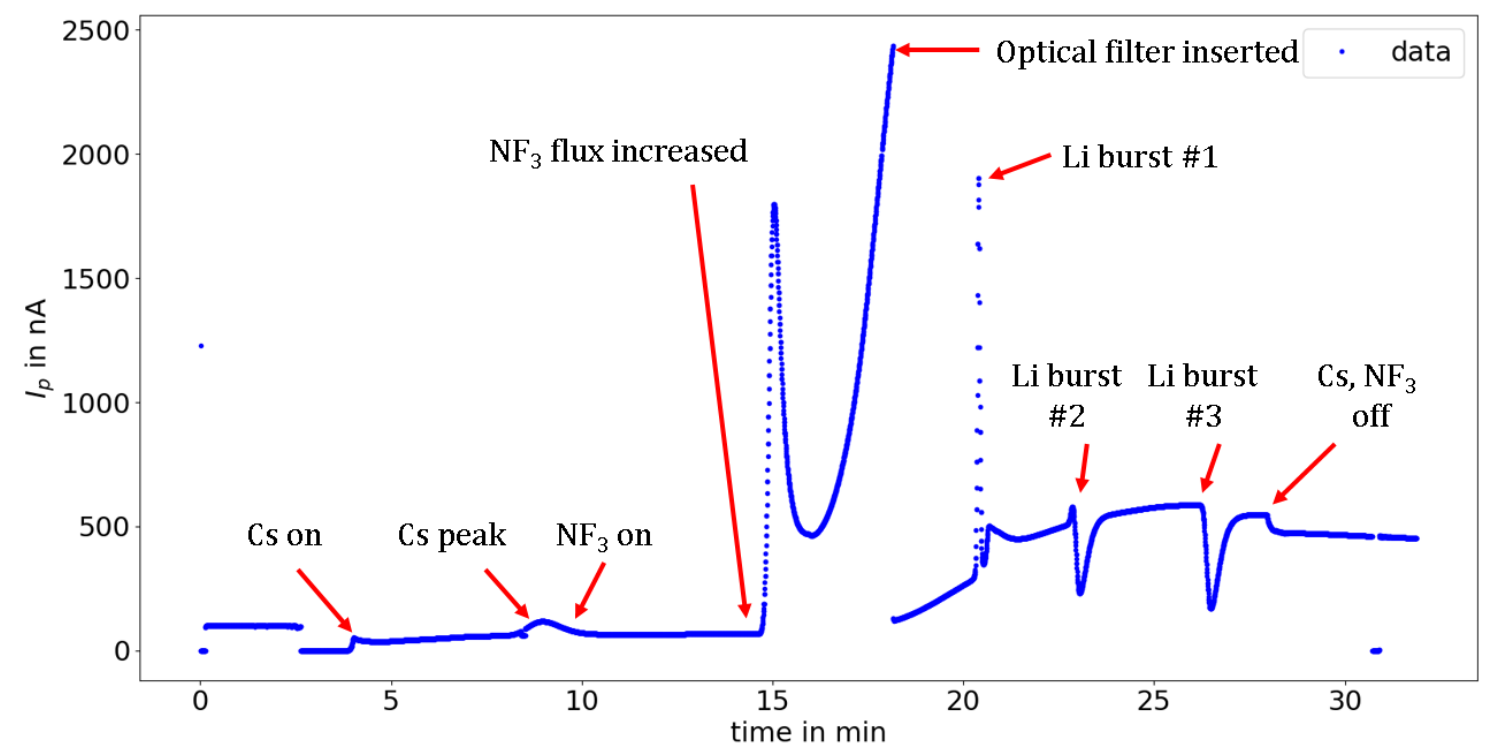

Figure 1: Activation sequence for GaAs: $\left(\mathrm{Cs}, \mathrm{Li}, \mathrm{NF}_{3}\right)$. A sharp peak of $I_{p}$ is visible directly after increasing the flux of $\mathrm{NF}_{3}$, followed by a short, but steep decrease before increasing steadily. This was probably caused by a brief imbalance of reaction products due to the slightly over-cesiated surface of the photo-cathode. A similar behavior can be observed during Li burst application.

\subsection{Results and Discussion}

The measured checkpoint values of $\eta$ are shown in tab. 1. It is notable that $\eta_{\text {ini }}$ is smaller for activation 1. QE scans showed that $\eta$ is highly dependent on laser-spot position, as $\eta_{\max }$ is significantly larger than $\eta_{\text {ini }}$, especially for activation 1 . This should be considered when measuring $\eta$ in the activation chamber and could be the cause of the apparently lower $\eta$ for Li-enhanced activation that has been reported in ref. [12]. Also, the difference between $\eta_{0}$ for activation 1 and activation 2 is significantly smaller compared to the same difference between corresponding $\eta_{\max }$. This may hint at a different strength of the Schottky effect and should be investigated further in future studies.

\begin{tabular}{lcccc}
\hline Act. method & Act. number & $\eta_{\text {ini }}$ in $\%$ & $\eta_{\max }$ in $\%$ & $\eta_{0}$ in $\%$ \\
\hline $\mathrm{Cs}, \mathrm{Li}_{\mathrm{NF}} \mathrm{NF}_{3}$ & 1 & $2.8 \pm 0.1$ & $4.84 \pm 0.05$ & $5.18 \pm 0.04$ \\
$\mathrm{Cs}, \mathrm{NF}_{3}$ & 2 & $3.1 \pm 0.1$ & $3.45 \pm 0.08$ & $4.59 \pm 0.05$ \\
\hline
\end{tabular}

Table 1: Quantum efficiency $\eta$ of bulk GaAs measured at different stages after activation and before beam extraction at $780 \mathrm{~nm}$. Measurements of $\eta$ have been taken with and without Li bursts in the co-deposition activation process immediately following activation $\left(\eta_{\text {ini }}\right)$, during a QE scan $\left(\eta_{\max }\right)$, and when starting beam extraction $\left(\eta_{0}\right)$.

Fig. 2 shows $\eta(t)$ for both runs. Run 1 consists of two parts: beam with $I=20 \mu \mathrm{A}$ was extracted for approximately $18 \mathrm{~h}$, before the beam current was increased to $I=100 \mu \mathrm{A}$. Run 2 started directly after activation with $I=100 \mu \mathrm{A}$. Fig. 3 compares $\eta$ of run 1 and run 2 for $I=100 \mu \mathrm{A}$, starting at $21 \mathrm{~h}$ after initial beam extraction for run 1 and at initial beam extraction for run 2 . Both 


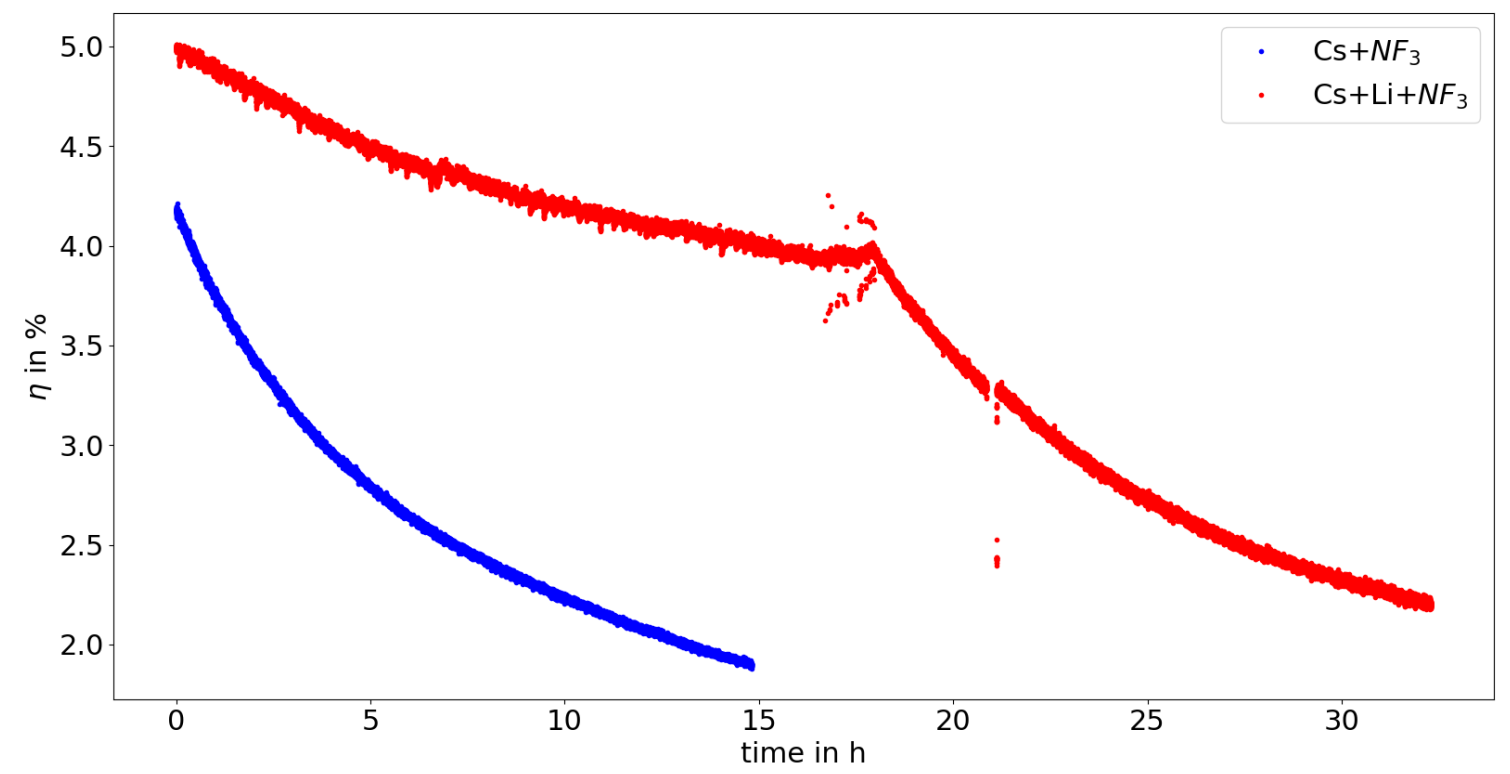

Figure 2: Comparison of $\eta(t)$ for GaAs:(Cs, $\left.\mathrm{Li}, \mathrm{NF}_{3}\right)$ (top, run 1) and GaAs:(Cs, $\left.\mathrm{NF}_{3}\right)$ (bottom, run 2). Run 1 was started with $I=20 \mu \mathrm{A}$ before continuing with $I=100 \mu \mathrm{A}$ after approximately $18 \mathrm{~h}$. The impact of increased beam current on the overall lifetime is clearly visible.

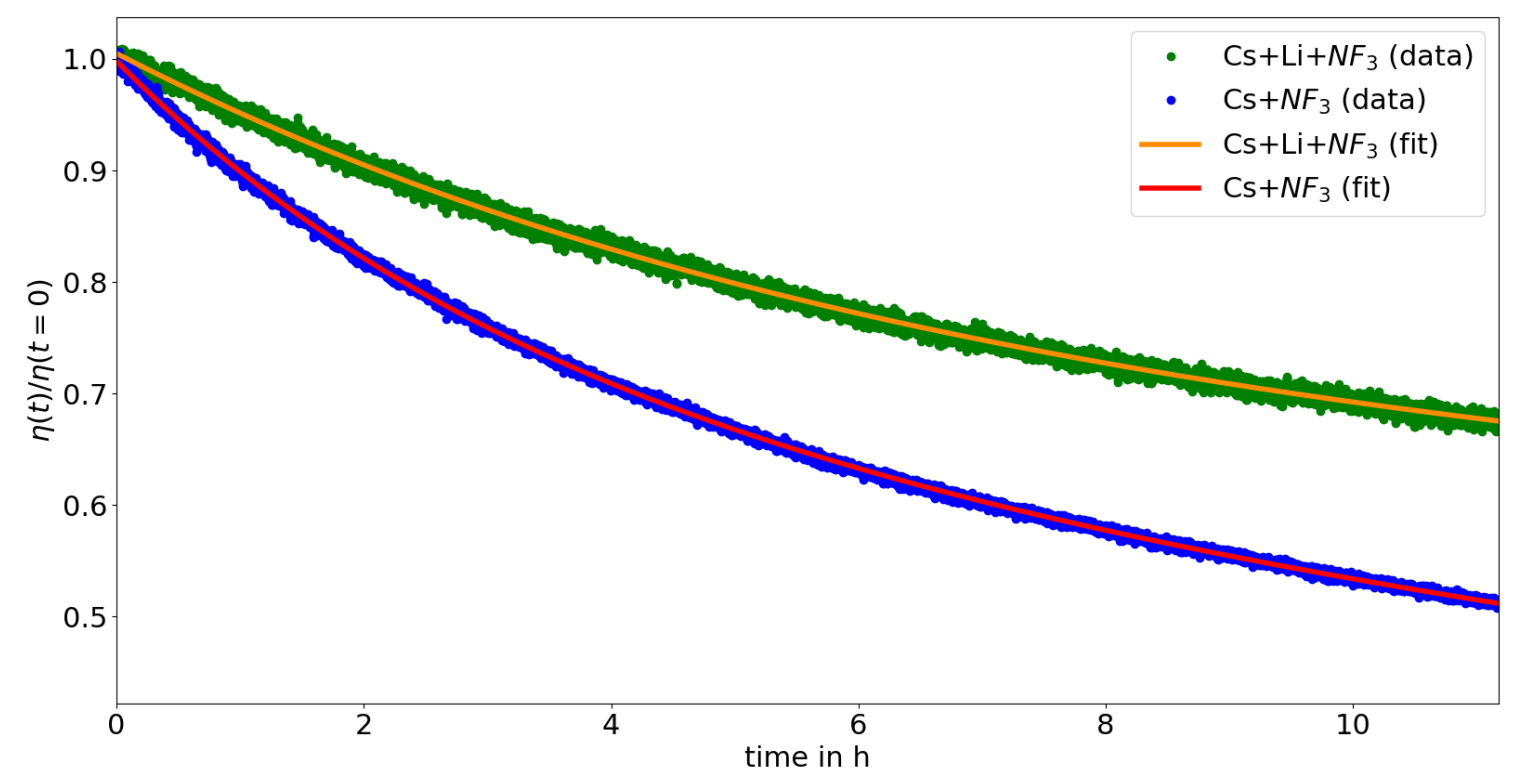

Figure 3: Normalized $\eta(t) / \eta(t=0)$ of GaAs:(Cs, $\left.\mathrm{Li}, \mathrm{NF}_{3}\right)$ (top, run 1) and GaAs: $\left(\mathrm{Cs}, \mathrm{NF}_{3}\right)$ (bottom, run 2), both with $I=100 \mu \mathrm{A}$, with corresponding bi-exponential fit. The Li-enhanced activation shows a slower rate of decay.

selected data sets were normalized on their respective first value $\eta(t=0)$. Run 1 displayed a slower rate of decay compared to run 2. However, a fit of eq. 2.2 as well as eq. 2.4a to the data with $I=100 \mu \mathrm{A}$ proved to be unsuccessful for both runs. Instead, a sum of two exponential decays or 
bi-exponential function [21]

$$
\eta(t)=\eta_{0} \cdot\left(\alpha \cdot e^{-t / \tau_{1}}+(1-\alpha) \cdot e^{-t / \tau_{2}}\right)
$$

with a weighting factor $0 \leq \alpha \leq 1$ and two independent decay constants $\tau_{1}$ and $\tau_{2}$, was used to obtain a matching fit to the data, as seen in fig. 3. The results are summarized in tab. 2. Estimates for $\tau_{c}$ and $k_{c}$, which were obtained from fitting eq. 2.5 to $\int_{0}^{t} I\left(t^{\prime}\right) d t^{\prime}$, are shown in tab. 3 .

\begin{tabular}{lccccc}
\hline Act. method & Act. number & $I$ in $\mu \mathrm{A}$ & $\tau_{1}$ in $\mathrm{h}$ & $\tau_{2}$ in $\mathrm{h}$ & weight. factor $\alpha$ \\
\hline $\mathrm{Cs}, \mathrm{Li}, \mathrm{NF}_{3}$ & 1 & 20 & $555 \pm 5$ & $9.1 \pm 0.1$ & $0.778 \pm 0.003$ \\
$\mathrm{Cs}, \mathrm{Li}_{1} \mathrm{NF}_{3}$ & 1 & 100 & $148 \pm 9$ & $6.47 \pm 0.07$ & $0.659 \pm 0.005$ \\
$\mathrm{Cs}, \mathrm{NF}_{3}$ & 2 & 100 & $33.96 \pm 0.03$ & $3.292 \pm 0.003$ & $0.6983 \pm 0.0002$ \\
\hline
\end{tabular}

Table 2: Experimental results obtained at UITF. Lifetimes obtained for $\mathrm{GaAs}:\left(\mathrm{Cs}, \mathrm{Li}, \mathrm{NF}_{3}\right)$ show an increase relative to GaAs: $\left(\mathrm{Cs}, \mathrm{NF}_{3}\right)$ at identical $I$, while the weighting factor $\alpha$ is slightly decreased. Extraction at lower $I$ showed the longest lifetimes and a higher $\alpha$.

\begin{tabular}{lcccc}
\hline Act. method & Act. number & $I$ in $\mu \mathrm{A}$ & $\tau_{c}$ in $\mathrm{h}$ & $k_{c}$ in $\mathrm{C}$ \\
\hline $\mathrm{Cs}, \mathrm{Li}, \mathrm{NF}_{3}$ & 1 & 20 & $50.84 \pm 0.03$ & $1.46 \pm 0.02$ \\
$\mathrm{Cs}, \mathrm{Li}, \mathrm{NF}_{3}$ & 1 & 100 & $26.71 \pm 0.01$ & $5.328 \pm 0.002$ \\
$\mathrm{Cs}, \mathrm{NF}_{3}$ & 2 & 100 & $17.67 \pm 0.01$ & $3.684 \pm 0.001$ \\
\hline
\end{tabular}

Table 3: Estimates for charge lifetime $\tau_{c}$ and extracted charge $k_{c}$, obtained from fitting eq. 2.5 to $Q(t)$. At identical $I$, both $\tau_{c}$ and $k_{c}$ show a significant increase for GaAs:(Cs, $\left.\mathrm{Li}, \mathrm{NF}_{3}\right)$ relative to GaAs:(Cs, $\left.\mathrm{NF}_{3}\right)$. At lower $I, \tau_{c}$ is even longer, while $k_{c}$ shows a significant decrease.

Each of the decay constants $\tau_{1}, \tau_{2}$, and $\tau_{c}$ show a significant increase for Li-enhanced run 1 compared to run 2. For $I=100 \mu \mathrm{A}$, run 1 yielded $\tau_{1}=148 \mathrm{~h}, \tau_{2}=6.4 \mathrm{~h}$ and $\tau_{c}=27 \mathrm{~h}$, while run 2 yielded $\tau_{1}=34 \mathrm{~h}, \tau_{2}=3.3 \mathrm{~h}$ and $\tau_{c}=18 \mathrm{~h}$. This replicates an increase in lifetime, which was observed at previous $\tau_{d}$ studies, under operational conditions. The extracted charge $k_{c}$ also shows an increase for Li-enhanced activation: for $I=100 \mu \mathrm{A}$, run 1 yielded $k_{c}=5.33 \mathrm{C}$ compared to $k_{c}=3.68 \mathrm{C}$ for run2. The first part of run 1 with $I=20 \mu \mathrm{A}$ yielded $k_{c}=1.46 \mathrm{C}$. This is about $27 \%$ of its value at $I=100 \mu \mathrm{A}$, with an expected ratio of about $20 \%$ due to the corresponding change of beam current. However, the ratio is higher since overall lifetime is increased for lower $I$.

The two decay constants $\tau_{1}$ and $\tau_{2}$ suggest that the photo-emission decay during beam production may be caused by two independent kinetic processes. However, they seem to match neither $\tau_{c}$ nor $\tau_{d}$ directly. Photo-emission decay measurements conducted without applied HV and without significant beam extraction, i.e. low $P_{L}$, can be described well with eq. 2.2. This decay is mainly dependent on vacuum conditions, which may be influenced by beam extraction. Gun chamber pressure was between $2.42 \times 10^{-11}$ mbar and $4.74 \times 10^{-11}$ mbar during run 1 , and between $1.98 \times 10^{-11}$ mbar and $2.66 \times 10^{-11}$ mbar during run 2 . A decrease in overall lifetime would be expected for run 1 due to a slightly higher pressure, but the opposite was observed. This hints at an enhancement of $\tau_{d}$ when Li is used. Important decay processes related to gun operation, such as ion back-bombardment and field emission, are directly linked to vacuum conditions, too: A higher 
pressure means that more ions can be produced and accelerated back to the cathode, and field emission increases pressure by releasing additional particles from the electrode and/or cathode surface. However, other decay processes such as thermal damage from laser illumination may cause a quantum efficiency decay that is not directly connected to vacuum conditions. Since both $\tau_{1}$ and $\tau_{2}$ increase with reduced $I$, one can assume that both of them depend on processes connected to beam extraction. The individual impact of both lifetimes and corresponding processes on the overall decay is represented by the weighting factor $\alpha$. For run 1, this factor showed a decrease for increased beam current, yielding $\alpha=0.78$ for $I=20 \mu \mathrm{A}$ and $\alpha=0.66$ for $I=100 \mu \mathrm{A}$. In Comparison, run 2 yielded $\alpha=0.7$ for $I=100 \mu \mathrm{A}$, showing a slight increase compared to the corresponding value of Li-enhanced run 1.

Also important is lifetime behavior with $I=$ const. and $P_{L} \neq$ const., since standard operation of a particle accelerator for experiments usually requires constant beam current. First studies on this topic have been conducted at UITF and are subject of ongoing evaluation. Additional investigations of operational lifetime are currently conducted at Photo-CATCH for both GaAs: $\left(\mathrm{Cs}, \mathrm{O}_{2}\right)$ and GaAs: $\left(\mathrm{Cs}, \mathrm{Li}, \mathrm{O}_{2}\right)$, aiming to reproduce the lifetime increase of Li-enhanced activation. Interestingly, a bi-exponential decay of $\eta$ has also been observed for $\mathrm{Cs}_{2}$ Te photo-cathodes [2]. Further detailed analysis is required in order to better understand how the different decay processes are connected and how they are represented by a bi-exponential decay model.

\section{Conclusion and Outlook}

World-first beam operation and $\tau_{c}$ measurements for GaAs: $\left(\mathrm{Cs}, \mathrm{Li}, \mathrm{NF}_{3}\right)$ have been successfully conducted at JLab, yielding an increase of $\eta_{0}$ by a factor of 1.13 and of $\tau_{c}$ by a factor of 1.5. The commonly used model of single exponential decay did not match the data. Instead, a bi-exponential model proved to describe the observed decay of the NEA layer well. This model yields two lifetime constants, the relation of which to $\tau_{c}$ and $\tau_{d}$ needs to be analysed in further investigations. Additional studies at both JLab and TU Darmstadt are planned to investigate operational behavior of both GaAs: $\left(\mathrm{Cs}, \mathrm{Li}, \mathrm{NF}_{3}\right)$ and $\mathrm{GaAs}:\left(\mathrm{Cs}, \mathrm{Li}, \mathrm{O}_{2}\right)$ and disentangle contribution of the dark and charge lifetimes.

\section{References}

[1] C.K. Sinclair, DC photoemission electron guns as ERL sources, Nucl. Instrum. Meth. A 557 (2006) 69-74 [DOI: 10.1016/j.nima.2005.10.053].

[2] T. Rao et al., Photocathodes for the energy recovery linacs, Nucl. Instrum. Meth. A 557 (2006) 124-130 [DOI: 10.1016/j.nima.2005.10.112].

[3] D. Abbot et al. (PEPPo collaboration), Production of Highly Polarized Positrons Using Polarized Electrons at MeV Energies, Phys. Rev. Lett. 116 (2016) 214801 [DOI:

10.1103/PhysRevLett.116.214801].

[4] A. Brachmann et al., The Polarized Electron Source for the International Collider (ILC) Project, AIP Conf. Proc. 915 (2007) 1091 [DOI: 10.1063/1.2750959].

[5] D.A. Orlov et al., Ultra Cold Photoelectron Beams for Ion Storage Rings, AIP Conf. Proc. 1149 (2009) 1007 [DOI: 10.1063/1.3215583]. 
[6] C. W. Leemann, D. R. Douglas, and G. A. Krafft, The Continuous Electron Beam Accelerator Facility: CEBAF at the Jefferson Laboratory, Annu. Rev. Nucl. Part. Sci. 51 (2001) 413-450 [DOI: 10.1146/annurev.nucl.51.101701.132327]

[7] C.K. Sinclair et al., Development of a high average current polarized electron source with long cathode operational lifetime, Phys. Rev. ST Accel. Beams 10 (2007) 023501 [DOI: 10.1103/PhysRevSTAB.10.023501].

[8] A. Freyberger, Commissioning and operation of $12 \mathrm{GeV} C E B A F$, in Proc. of IPAC'15, 1-5 (2015) [DOI: 10.18429/JACoW-IPAC2015-MOXGB2].

[9] G. Cheng et al., JLab New Injector Cryomodule Design, Fabrication and Testing, in Proc. of SRF'17, 158-162 (2017) [DOI: 10.18429/JACoW-SRF2017-MOPB045].

[10] X. Wei et al., Testing Frozen-Spin HD with electrons at Jefferson Lab - status update, in Proc. of PSTP'19, this conference (2019).

[11] N. Pietralla, The Institute of Nuclear Physics at the TU Darmstadt, Nuclear Physics News, 28:2 (2018) 4-11 [DOI: 10.1080/10619127.2018.1463013].

[12] N. Kurichiyanil et al., A test system for optimizing quantum efficiency and dark lifetime of GaAs photocathodes, JINST 14 (2019) P08025 [DOI: 10.1088/1748-0221/14/08/P08025].

[13] Y. Poltoratska et al., Status and recent developments at the polarized-electron injector of the superconducting Darmstadt electron linear accelerator S-DALINAC, J. Phys.: Conf. Ser. 298 (2011) 012002 [DOI: 10.1088/1742-6596/298/1/012002].

[14] K. Aulenbacher et al., Photocathode life time research at MAMI, in proc. of SPIN'04, World Scientific, Singapore, 975-979 (2005) [DOI: 10.1142/9789812701909_0196].

[15] C. Shonaka et al., A study of lifetime of GaAs photocathode for high brightness electron source, in proc. of PAC'09, 527-529 (2009).

[16] G.A. Mulhollan and J.C. Bierman, Enhanced chemical immunity for negative electron affinity GaAs photoemitters, J. Vac. Sci. Tech. A 26 (2008) 1195 [DOI: 10.1116/1.2965816].

[17] Y. Sun et al., The surface activation layer of GaAs negative electron affinity photocathode activated by Cs, Li, and $\mathrm{NF}_{3}$, Appl. Phys. Lett. 95 (2009) 174109 [DOI: 10.1063/1.3257730].

[18] P.A. Adderley et al., Load-locked dc high voltage GaAs photogun with an inverted-geometry ceramic insulator, Phys. Rev. ST Accel. Beams, 13 (2010) 010101 [DOI: 10.1103/PhysRevSTAB.13.010101].

[19] C. Hernandez-Garcia et al., Compact -300 kV dc inverted insulator photogun with biased anode and alkali-antimonide photocathode, Phys. Rev. Accel. Beams, 22 (2019) 113401 [DOI: 10.1103/PhysRevAccelBeams.22.113401].

[20] N. Kurichiyanil, Design and construction of a test stand for photocathode research and experiments, Technische Universität Darmstadt, Doctoral Dissertation (2017) [URN: urn:nbn:de:tuda-tuprints-59037].

[21] J. Navas et al., Evaluation of decay photocurrent measurements in dye-sensitized solar cells: Application to laser beam-induced current technique, Int. J. Energy Res., 36 (2012) 193-203 [DOI: 10.1002/er.1793]. 